\title{
ECONOMICS
}

\section{How Much Can the Swiss National Bank Contribute to the Financing of the Swiss Old Age Insurance (AHV)?}

\section{Ernst Juerg Weber}

Business School

The University of Western Australia 
How Much Can the Swiss National Bank Contribute to the Financing of the Swiss Old Age Insurance (AHV)?*

Wieviel kann die Schweizerische Nationalbank zur Finanzierung der AHV beitragen?

\author{
Ernst Juerg Weber \\ Senior Honorary Research Fellow \\ Department of Economics \\ University of Western Australia \\ Email: juerg.weber@uwa.edu.au
}

April 2019

* With the original German text and an English translation. 
The monetary base is the most important monetary policy tool of the Swiss National Bank (SNB) in order to achieve the statutory objective of price stability. The SNB can control the monetary base through the purchase and sale of gold, foreign exchange and domestic debt securities. A purchase of these assets leads to an increase in the monetary base and a sale to a reduction.

The attached balance sheet shows the assets acquired by the SNB as part of its monetary policy. At the end of 2017, the SNB owned gold for CHF 42.5 billion; CHF 794.6 billion in foreign exchange; and CHF 4 billion in securities denominated in Swiss francs. The foreign exchange position involves foreign financial assets, including debt securities and shares, while the Swiss franc securities include domestic and foreign debt instruments. The SNB is not active in the domestic stock market, as this would lead to undue state influence on Swiss companies.

The liabilities side of the balance sheet shows the financing of the SNB's investments. Banknotes in circulation amounted to CHF 81.6 billion at the end of 2017 and sight deposits held by domestic banks with the SNB totaled CHF 470.4 billion. Together this results in a supply of central bank money or monetary base of CHF 552 billion. Foreign liabilities, liabilities in foreign currency and the other sight liabilities are not added to the monetary base.

The surplus of assets over liabilities results in equity capital. At the end of 2017, the equity ratio of the SNB, which sets equity in relation to total assets, was 16.3 percent.

\section{The AHV Demography Fund}

The demographic development of Switzerland endangers the financing of the Swiss Old Age Insurance (AHV). For this reason, the Association for Faire Vorsorge, which was founded in 
$2017,{ }^{1}$ proposes that the SNB transfer part of its assets to a new AHV demography fund, the income of which could be used to finance the AHV. This raises the question as to whether the proposed fund of CHF 300 billion would unduly restrict the scope for monetary policy and would be financially acceptable to the SNB.

\section{Inflation}

Although the balance sheet of the SNB formally differs little from that of a commercial bank, there exists a fundamental disparity. A private bank or cantonal bank must convert demand or sight deposits into legal means of payment at the request of the depositor. This is not the case for the SNB as its banknotes are legal tender. Since the end of the gold standard and the abandonment of fixed exchange rates, the SNB has no longer been required to redeem its notes into gold or foreign currency. While a bank could convert deposits held with the SNB into banknotes, this would only be an exchange of liabilities on the SNB's balance sheet, with no consequences for its equity capital. Since the SNB creates legal tender in the form of banknotes, it can neither become illiquid nor go bankrupt.

With a central bank, however, inflation takes the place of bankruptcy. When the demand for central bank money falls and the central bank fails or refuses to convert the unwanted money into assets, the price level rises until the outstanding amount of central bank money equals the real value desired by domestic banks and the public. The SNB must sell assets to prevent inflation when the demand for central bank money falls. The Reichsbank could not stop the hyperinflation in Germany after the First World War because it ran out of usable assets. The same happened in Argentina, Zimbabwe and other countries with hyperinflation in recent times.

\footnotetext{
${ }^{1}$ https://www.fairevorsorge.ch/
} 
The demand for central bank money may decline for various reasons. The monetary base is subject to seasonal fluctuations and a decline in economic activity reduces the need for central bank money. A political crisis in Europe threatening Switzerland could lead to an escape from the Swiss franc, although this would be an unusual situation for Switzerland. However, a limited flight from the franc took place in the run-up to the Second World War, when people exchanged francs for gold. The SNB sold gold to redeem 15.5 percent of the monetary base from January 1939 to September 7, 1939. Another reason that the demand for central bank money could fall is competition from new means of payment. Cryptocurrencies have not yet met their high expectations, but the development of electronic payment technologies is certainly not yet complete.

The possibility that the demand for central bank money might decrease thus determines the stock of available assets of the SNB that is necessary for a stable currency. Figure 1 shows a monthly time series of the monetary base and Figure 2 shows the monthly changes in percent. Over the entire observation period, central bank money rose from CHF 5.8 billion in January 1950 to CHF 552.6 billion in September 2018. The average increase was 0.55 percent per month with a standard deviation of 5.1 percent. The largest increase in the monetary base was at 74.9 percent in August 2011 and the largest reduction was 17.8 percent in June 2010. So, for more than half a century, the monetary base has fallen never more than 17.8 percent per month or about three standard deviations, and the decline in central bank money just before the Second World War is in the same order of magnitude. However, these historical data do not provide information on the possible reduction in demand for Swiss central bank money in a future European crisis or in response to a successful cryptocurrency. 


\section{Is an AHV Demography Fund of CHF $\mathbf{3 0 0}$ billion Affordable for the SNB?}

At the end of 2017, the SNB held CHF 841.1 billion worth of assets available for monetary policy. This amount is determined by the balance of gold and foreign exchange, less current accounts held by foreign banks, other sight liabilities and foreign currency liabilities. The coverage ratio of the central bank money stock of CHF 552.1 billion thus amounted to 152.3 percent. It is this high coverage ratio of central bank money that is fueling the debate on financing the AHV by the SNB.

The proposed AHV Demographic Fund would have no impact on the SNB's equity capital, as the SNB would receive a debt certificate in return for the assets transferred that would be recorded in the balance sheet as an asset. However, this certificate would be completely illiquid and therefore could not be used for monetary policy. With a fund of CHF 300 billion, only CHF 541.1 billion worth of assets would be freely available for monetary policy, reducing the coverage ratio of the monetary base to 98 percent. Since the monetary base has never fallen more than 20 percent per month in the past, this would certainly be sufficient for the management of the monetary base in normal circumstances.

Even a coverage ratio of 100 percent does not, however, guarantee that there will be enough assets available when a crisis occurs. The SNB carries significant currency risk, as central bank money is denominated in francs and foreign exchange reserves are held in international currencies. The holdings of foreign shares also expose the SNB to stock market risk and bond holdings involve interest rate risk. Should the demand for Swiss central bank money suddenly fall, the SNB would have to sell its reserve assets at the prevailing market prices. If at that time the franc value of those assets should be low, the SNB might run out of assets. 
Another consideration concerns the stability of demand for central bank money. Switzerland benefits from a strong currency because there was always no doubt that the SNB has enough available assets to meet a fall in the demand for central bank money. Public confidence in the SNB's ability to pay led to the demand for central bank money never falling more than 20 percent per month, even in extraordinary times. However, reducing the coverage ratio of central bank money could undermine public confidence in the Swiss franc and enhance fluctuations in the demand for central bank money.

\section{Profit Distribution}

Instead of establishing a fund, the SNB could distribute part of its profits to the AHV. The advantage of this would be that no illiquid asset would weaken its balance sheet and the annual payments could be adjusted in the event of unforeseen changes in the financial position of the AHV or the SNB's ability to pay. Assuming a return of four percent, the SNB would have to distribute CHF 12 billion a year to the AHV to achieve the same level of financial support as the proposed fund of CHF 300 billion.

The profit of the SNB is subject to strong annual fluctuations. The SNB suffered major losses in 2013 and 2015 and generated high profits in 2014, 2016 and 2017. The profit of CHF 68.8 billion over these five years would have been just enough to cover the total dividend payments of CHF 6 million, the statutory distribution to the Swiss Confederation and Cantons totalling CHF 6.7 billion, and a new distribution in favour of the AHV of CHF 12 billion per year. This calculation shows that an AHV fund of CHF 300 billion, which equates to an annual payment of CHF 12 billion, would burden the SNB too much. However, an AHV fund of CHF 100 to 150 billion would be justifiable from the point of view of central bank solvency and monetary policy, as well as the SNB's long-term earning power. 
Figure 1

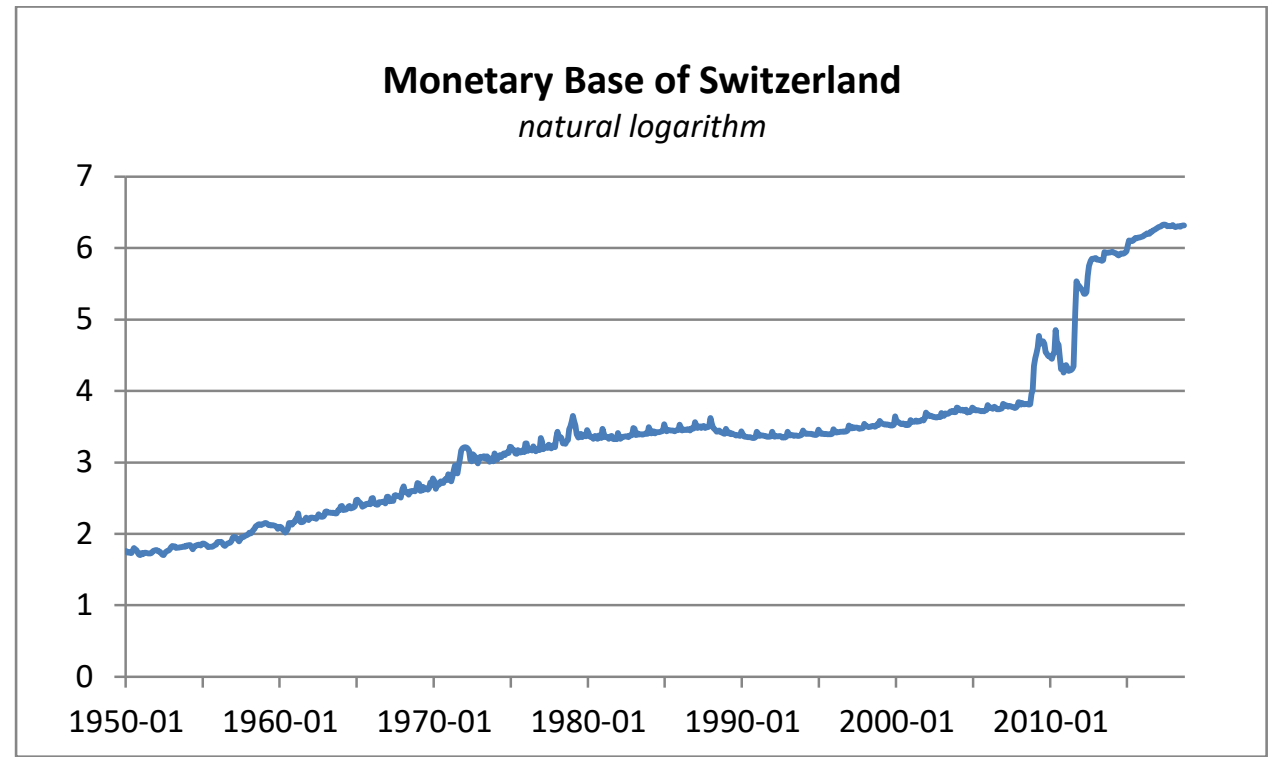

Source: SNB data portal

Figure 2

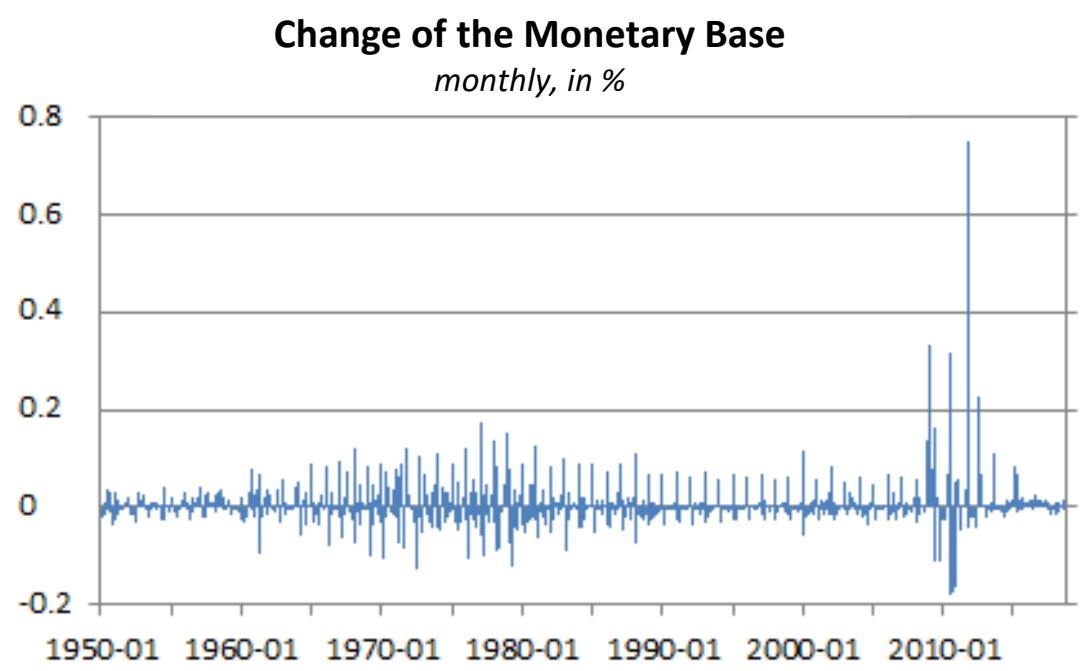




\section{Balance Sheet of the Swiss National Bank on 31 December 2017}

\begin{tabular}{|c|c|c|c|c|}
\hline \multicolumn{5}{|l|}{$\begin{array}{l}\text { ASSETS } \\
\text { In CHF millions }\end{array}$} \\
\hline & $\begin{array}{r}\text { Item } \\
\text { in Notes }\end{array}$ & 31.12 .2018 & 31.12 .2017 & Change \\
\hline Gold holdings & 01 & 42237.3 & 42494.0 & -256.7 \\
\hline Foreign currenoy investments & 02,25 & 763727.9 & 790124.8 & -26396.9 \\
\hline Reserve position in the IMF & 03,23 & 1188.4 & 871.3 & +317.1 \\
\hline International psyment instruments & 04,23 & 4440.6 & 4495.5 & -54.9 \\
\hline Monetary sssistance loans & 05,23 & 260.3 & 210.3 & +50.0 \\
\hline Claims from Swiss frano repo transactions & 22 & - & - & - \\
\hline Swiss frano securities & 06 & 3977.1 & 3956.2 & +20.9 \\
\hline Tangible assets & 07 & 435.5 & 396.3 & +39.2 \\
\hline Participations & 08,24 & 151.0 & 156.9 & -5.9 \\
\hline Other assets & 09,26 & 650.7 & 601.1 & +49.6 \\
\hline Total assets & & 817068.8 & 843306.4 & -26237.6 \\
\hline
\end{tabular}

\begin{tabular}{|c|c|c|c|c|}
\hline \multirow{2}{*}{$\begin{array}{l}\text { LIABILITIES } \\
\text { In CHF millions }\end{array}$} & & & & \\
\hline & $\begin{array}{r}\text { Item } \\
\text { in Notes }\end{array}$ & 31.12 .2018 & 31.12 .2017 & Change \\
\hline Banknotes in circulation & 10 & 82238.8 & 81638.9 & +599.9 \\
\hline Sight deposits of domestio banks & & 480634.3 & 470439.4 & +10194.9 \\
\hline Liabilities towards the Confederation & 11 & 15612.6 & 14754.8 & +857.8 \\
\hline Sight deposits of foreign banks and institutions & & 37101.7 & 54085.6 & -16983.9 \\
\hline Other sight liabilities & 12 & 41478.6 & 34398.8 & +7079.8 \\
\hline Liabilities from Swiss frano repo transactions & & - & - & - \\
\hline SNB debt certificates & & - & - & - \\
\hline Foreign currenoy liabilities & 13,25 & 34811.8 & 45933.6 & -11121.8 \\
\hline Counterpart of SDRs allocated by the IMF & 04 & 4486.5 & 4572.7 & -86.2 \\
\hline Other liabilities & 14,26 & 472.2 & 314.8 & +157.4 \\
\hline Equity & & & & \\
\hline Provisions for currenoy reserves' & & 67792.9 & 62771.2 & +5021.7 \\
\hline Share capital & 15 & 25.0 & 25.0 & - \\
\hline Distribution reserve' & & 67348.4 & 20000.0 & +47348.4 \\
\hline Annual result & & -14934.0 & 54371.6 & -69305.6 \\
\hline Total equity & & 120232.3 & 137167.8 & -16935.5 \\
\hline Total liabilities & & 817068.8 & 843306.4 & -26237.6 \\
\hline
\end{tabular}

Source: Annual Report of the SNB 


\section{Wieviel kann die Schweizerische Nationalbank zur Finanzierung der AHV beitragen?}

Die Regulierung der Notenbankgeldmenge ist das wichtigste Instrument der Schweizerischen Nationalbank (SNB), um das gesetzlich festgelegte Ziel der Preisstabilität zu erreichen. Die SNB kann die Notenbankgeldmenge über den Kauf und Verkauf von Gold, Devisen und inländischen Schuldpapieren steuern. Ein Kauf dieser Vermögenswerte führt zu einer Erhöhung der Notenbankgeldmenge und ein Verkauf zu einer Reduktion.

Die im Anhang angefügte Bilanz zeigt die von der SNB im Zuge der Geldpolitik erworbenen Vermögenswerte. Ende 2017 besass die SNB für CHF 42,5 Mrd. Gold; CHF 794,6 Mrd. Devisen und internationale Zahlungsmittel; und CHF 4 Mrd. auf Franken lautende Wertschriften. Bei den Devisen handelt es sich um ausländische Finanzaktiva einschliesslich Schuldpapiere und Aktien, während die Frankenwertschriften in- und ausländische Schuldpapiere umfassen. Die SNB ist nicht am inländischen Aktienmarkt tätig, da dies zu einer unzulässigen staatlichen Einflussnahme auf schweizerische Unternehmungen führen würde.

Die Passivseite der Bilanz zeigt die Finanzierung der von der SNB gehaltenen Vermögenswerte. Ende 2017 betrug der Banknotenumlauf CHF 81,6 Mrd. und die Giroguthaben der inländischen Banken beliefen sich auf CHF 470,4 Mrd.. Dies ergibt zusammen eine inländische Notenbankgeldmenge oder monetäre Basis von CHF 552 Mrd.. Die Auslandsverpflichtungen, die Verbindlichkeiten in Fremdwährung und die übrigen Sichtverbindlichkeiten werden der Notenbankgeldmenge nicht zugerechnet. 
Der Überschuss der Aktiven über die Verbindlichkeiten ergibt das Eigenkapital. Ende 2017 betrug die Eigenkapitalquote der SNB, die das Eigenkapital in Bezug zur Bilanzsumme setzt, 16,3 Prozent.

\section{Der AHV-Demografie-Fonds}

Die demografische Entwicklung der Schweiz gefährdet die Finanzierung der AHV. Aus diesem Grund schlägt der 2017 gegründete Verein Faire Vorsorge vor, ${ }^{2}$ dass die SNB einen Teil ihrer Vermögenswerte in einen neu zu errichtenden AHV-Demografie-Fonds überträgt, dessen Einkünfte zur Finanzierung der AHV verwendet werden könnten. Es stellt sich dabei die Frage, ob der vorgeschlagene Fonds von CHF 300 Mrd. den geldpolitischen Handlungsspielraum der SNB unzulässig einschränken würde und für die SNB finanziell tragbar wäre.

\section{Inflation}

Obwohl die Bilanz der SNB sich formal wenig von jener einer Handelsbank unterscheidet, besteht ein grundsätzlicher Gegensatz. Eine Privatbank oder Kantonalbank muss ihre Depositen auf Verlangen der Einleger in gesetzliche Zahlungsmittel umtauschen. Dies trifft für die SNB nicht zu, da ihre Banknoten gesetzliche Zahlungmittel sind. Seit dem Ende des Goldstandards und der Aufgabe fixer Wechselkurse ist die SNB nicht mehr verpflichtet, ihre Banknoten in Gold oder Devisen einzulösen. Eine Bank könnte zwar ihr Giroguthaben in Banknoten umtauschen, doch würde es sich dabei nur um einen Austausch von Passiven in der Bilanz der SNB handeln - ohne Konsequenzen für das ausgewiesene Eigenkapital. Da die SNB die gesetzlichen Zahlungsmittel schafft, kann sie weder illiquid werden noch in Konkurs gehen.

\footnotetext{
${ }^{2}$ https://www.fairevorsorge.ch/
} 
Bei einer Notenbank tritt jedoch die Inflation an die Stelle eines Konkurses. Wenn die Nachfrage nach Notenbankgeld fällt und die Notenbank ausserstande ist oder sich weigert, das unerwünschte Notenbankgeld in Vermögenswerte umzutauschen, steigt das Preisniveau, bis die umlaufende Notenbankgeldmenge dem von den inländischen Banken und dem Publikum gewünschten realen Wert entspricht. Die SNB muss Vermögenswerte verkaufen, um eine Währungsentwertung zu verhindern, wenn die Nachfrage nach Notenbankgeld fällt. Die Reichsbank konnte die Hyperinflation in Deutschland nach dem Ersten Weltkrieg nicht stoppen, weil ihr die verwertbaren Vermögenswerte ausgingen. Dasselbe geschah in Argentinien, Zimbabwe und in weiteren Ländern mit Hyperinflation in neuerer Zeit.

Die Nachfrage nach Notenbankgeld kann aus verschiedenen Gründen abnehmen. Die Notenbankgeldmenge ist saisonalen Schwankungen unterworfen und ein Rückgang der Wirtschaftstätigkeit verringert das Bedürfnis nach Notenbankgeld. Eine für die Schweiz bedrohliche politische Krise in Europa könnte zu einer Flucht aus dem Schweizerfranken führen, obwohl dies eine für die Schweiz ungewohnte Situation wäre. Eine begrenzte Flucht aus dem Schweizerfranken fand jedoch im Vorfeld des Zweiten Weltkrieges statt, als die Banken und das Publikum Franken gegen Gold eintauschten. Die Nationalbank verwendete Gold, um vom Januar 1939 bis zum 7. September 1939 15,5 Prozent der Notenbankgeldmenge zurückzuzahlen. Ein weiterer Grund, dass die Nachfrage nach Notenbankgeld fallen könnte, ist die Konkurrenz durch neue Zahlungsmittel. Die Kryptowährungen haben zwar bis jetzt die hoch gesteckten Erwartungen nicht erfüllt, die Entwicklung der elektronischen Zahlungstechnologie ist aber sicher noch nicht abgeschlossen. 
Die Möglichkeit, dass sich die Nachfrage nach Notenbankgeld verringert, bestimmt somit den für eine stabile Währung notwendingen Bestand an verfügbaren Vermögenswerten der SNB. Die Abbildung 1 enthält eine monatliche Zeitreihe der Notenbankgeldmenge und die Abbildung 2 zeigt die monatlichen Veränderungen gemessen in Prozent. Die Notenbankgeldmenge stieg über die gesamte Beobachtungsperiode von CHF 5,8 Mrd. im Januar 1950 auf CHF 552,6 Mrd. im September 2018. Die Zunahme betrug durchschnittlich 0,55 Prozent pro Monat mit einer Standardabweichung von 5,1 Prozent. Die grösste Steigerung der Notenbankgeldmenge erfolgte mit 74,9 Prozent im August 2011 und die grösste Reduktion betrug 17,8 Prozent im Juni 2010. Die Notenbankgeldmenge ist also seit mehr als einem halben Jahrhundert monatlich nie mehr als 17,8 Prozent oder etwa drei Standardabweichungen gefallen und der Rückgang der Notenbankgeldmenge kurz vor dem Zweiten Weltkrieg liegt in derselben Grössenordung. Diese Vergangenheitsdaten geben jedoch keine Auskunft über die mögliche Reduktion der Nachfrage nach schweizerischem Notenbankgeld in einer künftigen europäischen Krise oder als Reaktion auf eine erfolgreiche Kryptowährung.

\section{Ist ein AHV-Demografie-Fonds von CHF 300 Mrd. für die SNB tragbar?}

Die SNB hielt am Jahresende 2017 für CHF 841,1 Mrd. für die Geldpolitik verfügbare Vermögenswerte. Dieser Betrag ergibt sich aus dem in der Bilanz ausgewiesenen Bestand an Gold, Devisen und internationalen Zahlungsmitteln, abzüglich der Girokonten ausländischer Banken, übriger Sichtverbindlichkeiten und Verbindlichkeiten in Fremdwährung. Der Deckungsgrad der umlaufenden Notenbankgeldmenge von CHF 552,1 Mrd. belief sich somit auf 152,3 Prozent. Es ist dieser hohe Deckungsgrad der Notenbankgeldmenge, der die Debatte über die Finanzierung der AHV durch die SNB ausgelöst hat. 
Der vorgeschlagene AHV-Demografie-Fonds hätte keinen Einfluss auf das Eigenkapital der SNB, da sie für die abgegebenen Vermögenswerte eine Schuldverschreibung des Fonds erhielte, die in der Bilanz als Aktivum aufgeführt würde. Dieser Schuldtitel wäre aber völlig illiquid und könnte daher für die Geldpolitik nicht verwendet werde. Bei einem Fonds von CHF 300 Mrd. würden die für die Geldpolitik zur Verfügung stehenden Vermögenswerte noch CHF 541,1 Mrd. betragen und die Deckung der Notenbankgeldmenge würde auf 98 Prozent sinken. Da die Notenbankgeldmenge in der Vergangenheit monatlich nie mehr als 20 Prozent gefallen ist, wäre dies für die normale Steuerung der Notenbankgeldmenge sicher ausreichend.

Selbst ein Deckungsgrad von 100 Prozent garantiert jedoch nicht, dass im Krisenfall genügend Vermögenswerte zur Verfügung stehen. Die SNB trägt ein erhebliches Währungsrisiko, da die Notenbankgeldmenge eine Frankenverschuldung darstellt und sie ihre Devisen in verschiedenen Währungen hält. Bei den ausländischen Aktien der SNB besteht zudem das Risiko von Börsenverlusten und bei den Obligationen ein Zinsrisiko. Sollte die Nachfrage nach schweizerischem Notenbankgeld plötzlich sinken, müsste die SNB ihre Währungsreserven zu den vorherrschenden Marktpreisen verkaufen. Wenn zu diesem Zeitpunkt deren Marktwert tief sein sollte, könnten ihr dabei die Vermögenswerte ausgehen.

Eine weitere Überlegung betrifft die Stabilität der Nachfrage nach Notenbankgeld. Die Schweiz profitiert von einer starken Währung, weil es immer ausser Zweifel stand, dass die SNB genügend verfügbare Vermögenswerte besitzt, um eine inflationäre Entwicklung zu verhindern. Das Vertrauen der Marktteilnehmer in die Zahlungskraft der SNB bewirkte, dass die Notenbankgeldmenge auch in ausserordentlichen Zeiten monatlich nie mehr als 20 
Prozent fiel. Eine Verringerung der Deckung der Notenbankgeldmenge könnte die Marktteilnehmer jedoch verunsichern und zu einer Vergrösserung der Schwankungen in der Nachfrage nach Notenbankgeld führen.

\section{Gewinnverteilung}

Anstelle eines Fonds könnte die SNB einen Teil ihres Gewinnes an die AHV ausschütten. Der Vorteil davon wäre, dass kein illiquider Schuldtitel die Bilanz der SNB schwächen würde und die Auszahlungen bei unvorhergesehenen Veränderungen in der Finanzlage der AHV oder der Zahlungskraft der SNB angepasst werden könnten. Unter der Annahme einer Rendite von vier Prozent müsste die SNB jährlich CHF $12 \mathrm{Mrd}$. an die AHV ausschütten, um die gleiche Unterstützung der AHV wie der vorgeschlagene Fonds von CHF $300 \mathrm{Mrd}$. zu erreichen.

Der Gewinn der SNB ist starken jährlichen Schwankungen unterworfen. Die SNB erlitt grosse Verluste in den Jahren 2013 und 2015 und sie erwirtschaftete hohe Gewinne in 2014, 2016 und 2017. Das in diesen fünf Jahren angefallene ausschüttbare Ergebnis von CHF 68,8 Mrd. hätte für die Dividendenzahlungen von total CHF 6 Mio., die Ausschüttungen an den Bund und die Kantone von total CHF 6,7 Mrd. und eine neue Ausschüttung zu Gunsten der AHV von jährlich CHF 12 Mrd. knapp ausgereicht. Diese Rechnung zeigt, dass ein AHV Fonds von

CHF 300 Mrd., der bei einer Rendite von vier Prozent einer jährlichen Zahlung von CHF 12 Mrd. entspricht, die SNB zu stark belasten würde. Ein AHV Fonds von CHF 100 bis 150 Mrd. wäre aber sowohl aus der Sicht der Deckung des Notenbankgelds und der Geldpolitik als auch aus der Sicht der langfristigen Ertragskraft der SNB vertretbar. 
Abbildung 1

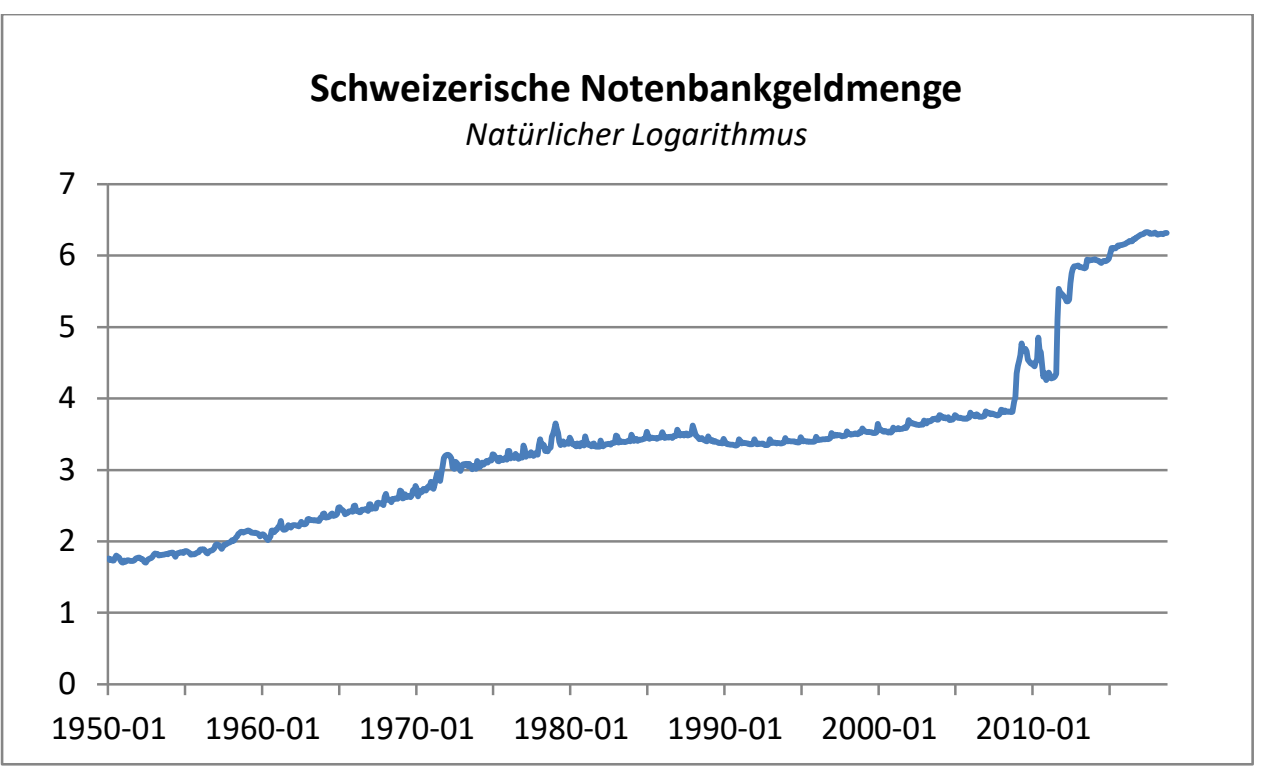

Quelle: Datenportal der SNB

Abbildung 2

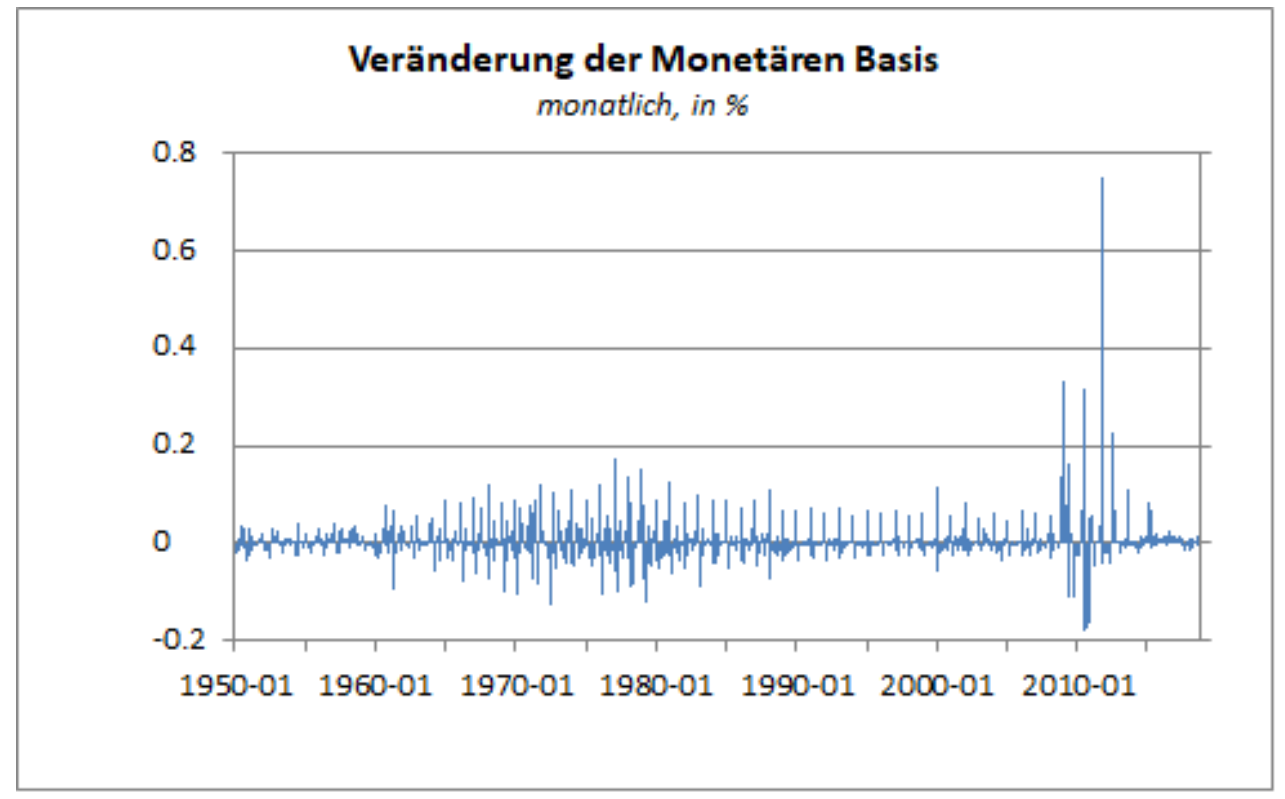


Bilanz der Schweizerischen Nationalbank per 31. Dezember 2017

\begin{tabular}{|c|c|c|c|c|}
\hline \multicolumn{5}{|l|}{$\begin{array}{l}\text { AKTIVEN } \\
\text { in Mio. Franken }\end{array}$} \\
\hline & $\begin{array}{r}\text { Ziffer } \\
\text { im Anhang }\end{array}$ & 31.12 .2017 & 31.12 .2016 & Veränderung \\
\hline Gold & 01 & 42494,0 & 39400,3 & $+3093,7$ \\
\hline Devisenanlagen & 02,25 & 790124,8 & 696104,2 & $+94020,6$ \\
\hline Reserveposition beim IWF & 03,23 & 871,3 & 1341,2 & $-469,9$ \\
\hline Internationale Zahlungsmittel & 04,23 & 4495,5 & 4406,2 & $+89,3$ \\
\hline Währungshilfekredite & 05,23 & 210,3 & 155,4 & $+54,9$ \\
\hline Forderungen aus Repogeschäften in Franken & 22 & - & - & - \\
\hline Wertschriften in Franken & 06 & 3956,2 & 3997,6 & $-41,4$ \\
\hline Sachanlagen & 07 & 396,3 & 375,1 & $+21,2$ \\
\hline Beteiligungen & 08,24 & 156,9 & 137,2 & $+19,7$ \\
\hline Sonstige Aktiven & 09,26 & 601,1 & 584,8 & $+16,3$ \\
\hline Total Aktiven & & 843306,4 & 746502,0 & $+96804,4$ \\
\hline
\end{tabular}

\section{PASSIVEN}

in Mio. Franken

\begin{tabular}{|c|c|c|c|c|}
\hline & $\begin{array}{r}\text { Ziffer } \\
\text { im Anhang }\end{array}$ & 31.12 .2017 & 31.12 .2016 & Veränderung \\
\hline Notenumlauf & 10 & 81638,9 & 78084,4 & $+3554,5$ \\
\hline Girokonten inländischer Banken & & 470439,4 & 468199,2 & $+2240,2$ \\
\hline Verbindlichkeiten gegenüber dem Bund & 11 & 14754,8 & 7229,7 & $+7525,1$ \\
\hline Girokonten ausländischer Banken und Institutionen & & 54085,6 & 24585,0 & $+29500,6$ \\
\hline Übrige Sichtverbindlichkeiten & 12 & 34398,8 & 30035,6 & $+4363,2$ \\
\hline Verbindlichkeiten aus Repogeschäften in Franken & & - & - & - \\
\hline Eigene Schuldverschreibungen & & - & - & - \\
\hline Verbindlichkeiten in Fremdwährungen & 13,25 & 45933,6 & 49096,3 & $-3162,7$ \\
\hline Ausgleichsposten für vom IWF zugeteilte SZR & 04 & 4572,7 & 4492,8 & $+79,9$ \\
\hline Sonstige Passiven & 14,26 & 314,8 & 251,6 & $+63,2$ \\
\hline \multicolumn{5}{|l|}{ Eigenkapital } \\
\hline Rückstellungen für Währungsreserven ${ }^{1}$ & & 62771,2 & 58121,5 & $+4649,7$ \\
\hline Aktienkapital & 15 & 25,0 & 25,0 & - \\
\hline Ausschüttungsreserve ${ }^{1}$ & & 20000,0 & 1904,5 & $+18095,5$ \\
\hline Jahresergebnis & & 54371,6 & 24476,4 & $+29895,2$ \\
\hline Total Eigenkapital & & 137167,8 & 84527,4 & $+52640,4$ \\
\hline Total Passiven & & 843306,4 & 746502,0 & +96804,4 \\
\hline
\end{tabular}

Quelle: Jahresbericht der SNB 
Editor, UWA Economics Discussion Papers:

Sam Hak Kan Tang

University of Western Australia

35 Sterling Hwy

Crawley WA 6009

Australia

Email: ecoadmin@biz.uwa.edu.au

The Economics Discussion Papers are available at:

Since 1980: $\quad$ https://ideas.repec.org/s/uwa/wpaper.html

Since 2004: http://www.business.uwa.edu.au/school/disciplines/economics

\begin{tabular}{|c|c|c|}
\hline \multicolumn{3}{|c|}{ ECONOMICS DISCUSSION PAPERS - 2016} \\
\hline $\begin{array}{l}\text { DP } \\
\text { NUMBER }\end{array}$ & AUTHORS & TITLE \\
\hline 16.01 & Xu, R., Wu, Y. and Luan, J. & $\begin{array}{l}\text { ANALYSIS OF FARMERS’ WILLINGNESS TO ADOPT } \\
\text { GENETICALLY MODIFIED INSECT-RESISTANT RICE IN } \\
\text { CHINA }\end{array}$ \\
\hline 16.02 & $\begin{array}{l}\text { Lia, Y., Fan, J., Zhao, D., Wu, Y. and } \\
\text { Li, J. }\end{array}$ & $\begin{array}{l}\text { TIERED GASOLINE PRICING: A PERSONAL CARBON } \\
\text { TRADING PERSPECTIVE }\end{array}$ \\
\hline 16.03 & Clements, K.W., Lan, Y. and Si, J. & UNCERTAINTY IN CURRENCY MISPRICING \\
\hline 16.04 & Parsons, C. and Vézina, P.L. & $\begin{array}{l}\text { MIGRANT NETWORKS AND TRADE:THE VIETNAMESE } \\
\text { BOAT PEOPLE AS A NATURAL EXPERIMENT }\end{array}$ \\
\hline 16.05 & Chang, S., Connelly, R. and Ma, P. & $\begin{array}{l}\text { WHAT WILL YOU DO IF I SAY 'I DO’?: THE EFFECT OF } \\
\text { THE SEX RATIO ON TIME USE WITHIN TAIWANESE } \\
\text { MARRIED COUPLES }\end{array}$ \\
\hline 16.06 & Yu, F. and Wu, Y. & $\begin{array}{l}\text { BIASES IN PATENT EXAMINATION AND FIRMS’ } \\
\text { RESPONSES: EVIDENCE FROM THE } \\
\text { PHARMACEUTICAL INDUSTRY }\end{array}$ \\
\hline 16.07 & $\begin{array}{l}\text { Fan, J., Li, J., Wu, Y., Wang, S. and } \\
\text { Zhao, D. }\end{array}$ & $\begin{array}{l}\text { THE EFFECTS OF ALLOWANCE PRICE ON ENERGY } \\
\text { DEMAND UNDER A PERSONAL CARBON TRADING } \\
\text { SCHEME }\end{array}$ \\
\hline 16.08 & Golley, J., Tyers, R. and Zhou, Y. & $\begin{array}{l}\text { CONTRACTIONS IN CHINESE FERTILITY AND } \\
\text { SAVINGS: LONG RUN DOMESTIC AND GLOBAL } \\
\text { IMPLICATIONS }\end{array}$ \\
\hline 16.09 & McGrath, G. and Neill, K. & $\begin{array}{l}\text { FOREIGN AND DOMESTIC OWNERSHIP IN WESTERN } \\
\text { AUSTRALIA'S GAS MARKET }\end{array}$ \\
\hline 16.10 & Clements, K.W. and Si, J. & SIMPLIFYING THE BIG MAC INDEX \\
\hline 16.11 & Priyati, R.Y. and Tyers, R. & $\begin{array}{l}\text { PRICE RELATIONSHIPS IN VEGETABLE OIL AND } \\
\text { ENERGY MARKETS }\end{array}$ \\
\hline 16.12 & Wu, J., Wu, Y. and Wang, B. & $\begin{array}{l}\text { THE GREENNESS OF CHINESE CITIES: CARBON } \\
\text { DIOXIDE EMISSION AND ITS DETERMINANTS }\end{array}$ \\
\hline 16.13 & $\begin{array}{l}\text { Arslan, C., Dumont, J.C., Kone, Z., } \\
\text { Özden, Ç., Parsons, C. and } \\
\text { Xenogiani, T. }\end{array}$ & $\begin{array}{l}\text { INTERNATIONAL MIGRATION TO THE OECD IN THE } \\
\text { TWENTY-FIRST CENTURY }\end{array}$ \\
\hline 16.14 & Tomioka, K. and Tyers, R. & $\begin{array}{l}\text { HAS FOREIGN GROWTH CONTRIBUTED TO } \\
\text { STAGNATION AND INEQUALITY IN JAPAN? }\end{array}$ \\
\hline 16.15 & Donovan, J. and Hartley, P. & RIDING THE IRON ORE CYCLE: ACTIONS OF \\
\hline
\end{tabular}




\begin{tabular}{|l|l|l|}
\hline 16.16 & Czaika, M. and Parsons, C. & $\begin{array}{l}\text { AUSTRALIA'S MAJOR PRODUCERS } \\
\text { HIGH-SKILLED MIGRATION IN TIMES OF GLOBAL } \\
\text { ECONOMIC CRISIS }\end{array}$ \\
\hline 16.17 & Lefroy, T., Key, J. and Kingwell, R. & $\begin{array}{l}\text { A LONGITUDINAL EXAMINATION OF BROADACRE } \\
\text { FARM SIZE AND PERFORMANCE IN WESTERN } \\
\text { AUSTRALIA }\end{array}$ \\
\hline 16.18 & Arthmar, R. and McLure, M. & $\begin{array}{l}\text { SRAFFA, MYRDAL AND THE 1961 SÖDERSTRÖM GOLD } \\
\text { MEDAL }\end{array}$ \\
\hline 16.19 & Azwar, P. and Tyers, R. & $\begin{array}{l}\text { POST-GFC EXTERNAL SHOCKS AND INDONESIAN } \\
\text { ECONOMIC PERFORMANCE }\end{array}$ \\
\hline 16.20 & Chen, A. and Groenewold, N. & $\begin{array}{l}\text { OUTPUT SHOCKS IN CHINA: DO THE DISTRIBUTIONAL } \\
\text { EFFECTS DEPEND ON THE REGIONAL SOURCE? }\end{array}$ \\
\hline 16.21 & Wu, Y., Zhu, X. and Groenewold, N. & $\begin{array}{l}\text { THE DETERMINANTS AND EFFECTIVENESS OF } \\
\text { INDUSTRIAL POLICY IN CHINA: A STUDY BASED ON } \\
\text { FIVE-YEAR PLANS }\end{array}$ \\
\hline 16.22 & Liu, H. & $\begin{array}{l}\text { THE INCOME AND PRICE SENSITIVITY OF DIETS } \\
\text { GLOBALLY }\end{array}$ \\
\hline 16.23 & Asano, A., Neill, K. and Yamazaki, S. & $\begin{array}{l}\text { DECOMPOSING FISHING EFFORT: MODELLING THE } \\
\text { SOURCES OF INEFFICIENCY IN A LIMITED-ENTRY } \\
\text { FISHERY }\end{array}$ \\
\hline 16.24 & Golley, J., Tyers, R. and Zhou, Y. & $\begin{array}{l}\text { FERTILITY AND SAVINGS CONTRACTIONS IN CHINA: } \\
\text { LONG-RUN GLOBAL IMPLICATIONS }\end{array}$ \\
\hline 16.25 & Taylor, G., Tyers, R. & $\begin{array}{l}\text { SECULAR STAGNATION: DETERMINANTS AND } \\
\text { CONSEQUENCES FOR AUSTRALIA }\end{array}$ \\
\hline
\end{tabular}

\begin{tabular}{|c|c|c|}
\hline \multicolumn{3}{|c|}{ ECONOMICS DISCUSSION PAPERS - 2017} \\
\hline $\begin{array}{c}\text { DP } \\
\text { NUMBER }\end{array}$ & AUTHORS & TITLE \\
\hline 17.01 & Tyers, R. and Zhou, Y. & $\begin{array}{l}\text { AUTOMATION AND INEQUALITY WITH TAXES AND } \\
\text { TRANSFERS }\end{array}$ \\
\hline 17.02 & Ye, L. and Robertson, P. & $\begin{array}{l}\text { HITTING THE GREAT WALL: RURAL-URBAN } \\
\text { MIGRATION AND CHINA'S GROWTH SLOWDOWN }\end{array}$ \\
\hline 17.03 & Ye, L. and Robertson, P. & $\begin{array}{l}\text { MIGRATION AND GROWTH IN CHINA: A SCEPTICAL } \\
\text { ASSESSMENT OF THE EVIDENCE }\end{array}$ \\
\hline 17.04 & Clements, K. Si, J. and Vo, L. & $\begin{array}{l}\text { FOOD AND AGRICULTURAL PRICES ACROSS } \\
\text { COUNTRIES AND THE LAW OF ONE PRICE }\end{array}$ \\
\hline 17.05 & $\begin{array}{l}\text { Chen, M., Clements, K., Gao, G. and } \\
\text { Si, J. }\end{array}$ & THREE FACTS ABOUT WORLD METAL PRICES \\
\hline 17.06 & $\begin{array}{l}\text { Cornes, R., Fiorini, L. and } \\
\text { Maldonado, W. }\end{array}$ & $\begin{array}{l}\text { EXPECTATIONAL STABILITY IN AGGREGATIVE } \\
\text { GAMES }\end{array}$ \\
\hline 17.07 & Hartley, P. & $\begin{array}{l}\text { THE COST OF DISPLACING FOSSIL FUELS: SOME } \\
\text { EVIDENCE FROM TEXAS }\end{array}$ \\
\hline 17.08 & Shehabi, M. R. & ASSESSING KUWAITI ENERGY PRICINING REFORMS \\
\hline 17.09 & Perdana, S. and Tyers, R. & $\begin{array}{l}\text { GLOBAL CLIMATE CHANGE MITIGATION: STRATEGIC } \\
\text { INTERACTION OR UNILATERAL GAINS? }\end{array}$ \\
\hline 17.10 & McLure, M. & $\begin{array}{l}\text { RICARDIAN EQUIVALENCE, THE ITALIAN FISCAL } \\
\text { TRADITION AND WA GOVERNMENT NET DEBT }\end{array}$ \\
\hline 17.11 & Trinh, J. & $\begin{array}{l}\text { THE PARETO DISTRIBUTION AND ITS RELATIONSHIP } \\
\text { TO PIKETTY'S THIRD FUNDAMENTAL LAW OF } \\
\text { CAPITALISM }\end{array}$ \\
\hline 17.12 & McLure, M. & THINKING OUTSIDE THE BOX: A NEW HISTORY OF \\
\hline
\end{tabular}




\begin{tabular}{|c|c|c|}
\hline & & $\begin{array}{l}\text { EDGEWORTH'S AND PARENTO'S DEVELOPMENT OF } \\
\text { THE BOX DIAGRAM }\end{array}$ \\
\hline 17.13 & Zhou, Y. and Tyers, R. & AUTOMATION AND INEQUALITY IN CHINA \\
\hline 17.14 & Arthmar, R. and McLure, M. & $\begin{array}{l}\text { THE ECONOMIC AND SOCIAL CONSEQUENCES OF THE } \\
\text { WAR: PIGOU, THE PRESS AND THE STRUGGLE FOR AN } \\
\text { HONOURABLE PEACE }\end{array}$ \\
\hline 17.15 & Shehabi, M. R. & $\begin{array}{l}\text { CONTEMPORARY KUWAITISATION DYNAMICS AND } \\
\text { THEIR HISTORICAL PERSPECTIVES }\end{array}$ \\
\hline 17.16 & Clements, K. and Si, J. & $\begin{array}{l}\text { WHAT DO AUSTRALIAN ECONOMICS PhDs DO? THE } \\
\text { UWA EXPERIENCE }\end{array}$ \\
\hline 17.17 & Arthmar, R. and McLure, M. & KEYNES AND THE ROYAL SWEDISH ACADEMY \\
\hline 17.18 & Chen, A. and Groenewold, N. & $\begin{array}{l}\text { CHINA’S ‘NEW NORMAL’: IS THE GROWTH } \\
\text { SLOWDOWN DEMAND- OR SUPPLY- DRIVEN? }\end{array}$ \\
\hline 17.19 & Groenewold, N. & $\begin{array}{l}\text { CHINA'S ‘NEW NORMAL’: HOW WILL CHINA’S } \\
\text { GROWTH SLOWDOWN AFFECT AUSTRALIA'S } \\
\text { GROWTH? }\end{array}$ \\
\hline
\end{tabular}

ECONOMICS DISCUSSION PAPERS - 2018

\begin{tabular}{|c|c|c|}
\hline $\begin{array}{c}\text { DP } \\
\text { NUMBER }\end{array}$ & AUTHORS & TITLE \\
\hline 18.01 & Tyers, R. and Zhou, Y. & LOST INFLATION? \\
\hline 18.02 & McLure, M. & PARETIAN FISCAL SOCIOLOGY \\
\hline 18.03 & McLure, M. and Montesano, A. & $\begin{array}{l}\text { THINKING OUTSIDE THE BOX: EDGEWORTH, PARETO } \\
\text { AND THE EARLY HISTORY OF THE BOX DIAGRAM }\end{array}$ \\
\hline 18.04 & Tyers, R. and Zhou, Y. & DEFLATION FORCES AND INEQUALITY \\
\hline 18.05 & Clements, K. and Si, J. & DIVISIA AND FRISCH ARE FRIENDS \\
\hline 18.06 & Trinh, J. and McLure, M. & $\begin{array}{l}\text { PILETTY'S }(\mathrm{r}-\mathrm{g}) \text { LAW IS PARETO'S LAW: CONSISTENT } \\
\text { ANALYSES OF INCOME DISTRIBUTION PREDICATED ON } \\
\text { INCONSISTENT DEFINITIONS OF INEQUALITY }\end{array}$ \\
\hline 18.07 & Tyers, R. and Zhou, Y. & $\begin{array}{l}\text { AUTOMATION, TAXES AND TRANSFERS } \\
\text { WITH INTERNATIONAL RIVALRY }\end{array}$ \\
\hline 18.08 & Li, Q., Vo, L. H. and Wu, Y. & INTANGIBLE CAPITAL DISTRIBUTION IN CHINA \\
\hline 18.09 & Vo, L. H. & $\begin{array}{l}\text { WHY DON'T AGRICULTURAL PRICES ALWAYS ADJUST } \\
\text { TOWARDS PARITY? }\end{array}$ \\
\hline 18.10 & Magnusson, L. M. and Tarverdi, Y. & MEASURING GOVERNANCE: WHY DO ERRORS MATTER? \\
\hline
\end{tabular}

